\title{
MONITORING PHOTOVOLTAIC SYSTEMS LEVERAGING BIG DATA INFRASTRUCTURE
}

\author{
Ștefan PREDA \\ Bucharest University of Economic Studies, Romania \\ spreda2003@yahoo.com \\ Adela BÂRA \\ Bucharest University of Economic Studies, Romania \\ bara.adela@ie.ase.ro
}

\begin{abstract}
Photovoltaic (PV) systems require advanced monitoring and control activities for a stable operation during time. To be accurate, monitoring activities should be based on large volumes of data collected from the PV system by means of inverters, PV modules sensors, weather stations or web sites. In most cases data are coming continuous, in streaming and in high volumes and, for monitoring purposes, big data technologies are considered for processing and storage.
\end{abstract}

Keywords: Photovoltaic systems, Big Data, monitoring, optimization, streaming JEL classification: L86, D80, Q40.

DOI: $10.12948 / \mathrm{ie} 2019.03 .04$

\section{Introduction}

In the past, data regarding the operation of the PV system was collected only from the inverter. This represents the most accessible way to monitor the PV system by checking the inverter output. Some inverters have the possibility to send over the internet detailed data about generated power, representing through graphs the generated power over time, together with summary data about generated power per time unit (hour, days, weeks, months, year). This represents an accessible way to check the operation of the system. In case the PV system has few inverters, then the solution for monitoring and control is not so complex. But, in case of a PV system with many inverters or a distributed PV-PP, even with summarized data provided by each inverter, processing and aggregating the available data require more complex solutions and technologies.

Once with hardware improvements, the low price and the availability of different types of sensors [1], data is now collected from the PV panels. This leads to an increased volume of data coming in streaming from the multitude of PV sensors.

PV systems monitoring also depends on PV technology (crystalline silicon CIGS, crystalline silicon) and, to overcome the differences, the authors in [2] proposed the use of linear regressions plots between two variables of the PV system. In real situation those plots are straight lines, relationship is repeating periodically, comparing to the historical lines and the observation of some significant changes between appropriate time intervals is a potential indicator that system behaviour is not normal. From this perspective, monitoring is based on time frame comparison of the PV parameters.

To increase the monitoring accuracy, in a PV power plant (PV PP) different type of sensors can be installed generating huge amount of data, depending on the time resolution and the size of the PV system. In case of distributed PV PP, the complexity of acquiring data from sensors is also increased. To manage the data processing and storage issues, big data technologies like 
Kafka and Hadoop comes into place. In [5] the authors proposed a method for analysing sensors data from IoT devices, leveraging Hadoop features and, since there is a similarity between collecting data from sensors for PV systems monitoring and IoT devices, the proposed method can be also applied in case of large PV-PP.

The main objective of the PV systems monitoring is to determine malfunctions or possible failures, to determine in advance if a component of the system is damaged or to prevent future losses and failures (preventive maintenance). Mainly, the monitoring of the PV system should include the following:

- inverter output power loss;

- inverter malfunctioning or failure;

- problems regarding the operation of the PV panels;

- connectivity problems between the inverter and the PV panels or batteries (in case they are installed);

- connectivity problems between the PV system and the main grid for on-grid systems.

The most relevant measure in PV system monitoring is the generated power for which three challenges are encountered:

1. The system output depends on the meteorological conditions that represents the most important factor for generation. To estimate (forecast) this input factor is the most challengeable aspect. The other input parameters are related to the status of the PV system components such as inverters and PV modules;

2. The generated power decreases over time due to the aging of the PV modules (technological issues) and decrease of the PV system yield;

3. The generated power depends on the solar irradiation and sun position, that leads to a Gaussian distribution during the day.

The values of these parameters and corresponding generated power need to be stored for historical comparison in order to estimate the generated power and monitor the PV system operation with high accuracy. Thus, in case significant differences are recorded for the generated power in similar conditions then certain issues related to the PV components should be further investigated.

The paper proposes an architecture for monitoring, processing and storage of the PV system parameters and provide a method for investigating the operation condition over time by comparing these input parameters. Also, a prototype of this solution is presented at the end of the paper.

\section{The big data architecture for photovoltaic monitoring}

The proposed architecture for monitoring the PV system leveraging big data technologies contains several components (blocks) to support the monitoring issues (Figure 1).

The architecture leverages classical big data architectures presented in [3], [4] referring to blocks like Storage \& Streams, Resource management, Processing engines, basically known as big data framework that uses technologies to implement each block. For example, for processing engine several technologies such MapReduce, Spark, Storm or Flink can be used. The Input Data Block is a particular case for the PV systems. In this case data is collected from different sources such as: PV modules sensors or loggers, weather sensors of web sites. Data can be collected at specific time intervals (10 to 60 minutes) or it can be collected continuously, in streaming by Kafka or Flume which may send data to Hadoop for storage in HDFS. 
www.conferenceie.ase.ro

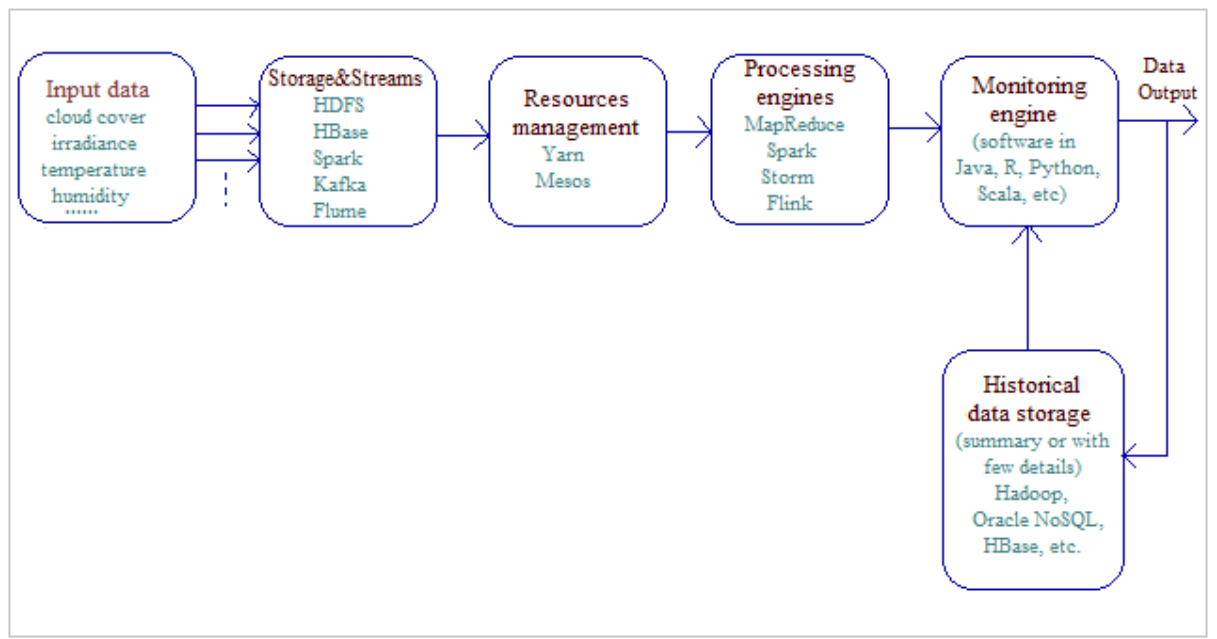

Figure 1. The monitoring architecture based on big data technologies

The Monitoring Engine will check data received from the streaming process or retrieved from the HDFS files and will compare the actual values with historical records to check the PV system operating conditions. Sample logical flow performed by the Monitoring Engine is synthetized in Figure 2. For comparison purposes, the Monitoring Engine will display the output on graphs or series of values.

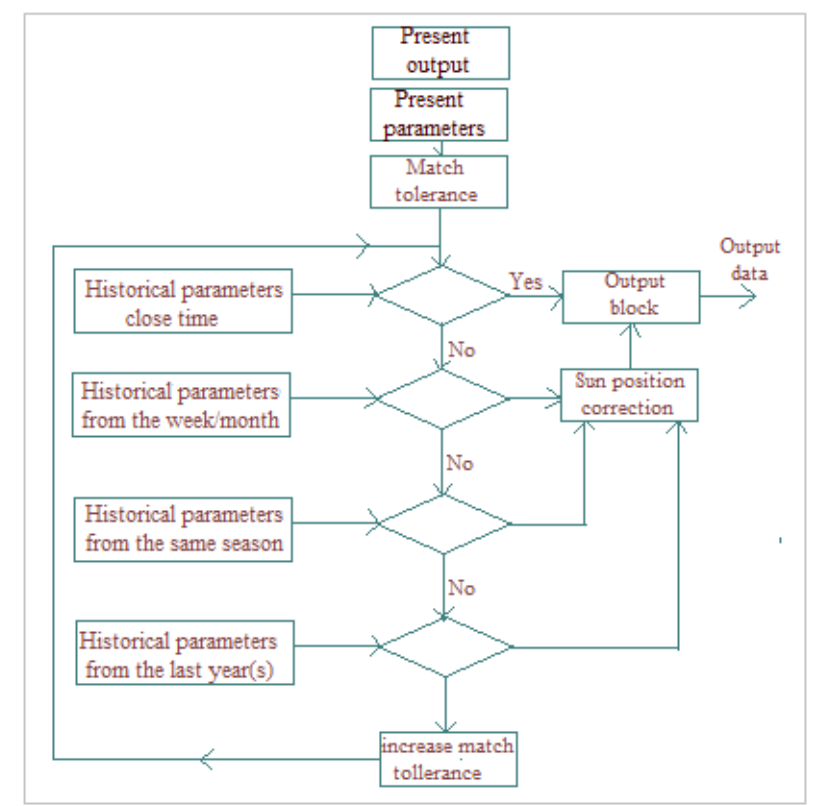

Figure 2. The processing flow performed by the Monitoring Engine

The Monitoring Engine receives as input the actual (current) generated power correlated with the input parameters collected from weather sensors and PV modules sensors or loggers. The most significant weather parameters, according to [6], [7] are the sun irradiance, temperature, sky cloud cover, wind speed and wind direction.

First, it checks if the actual records are similar with historical records from closer time periods, with a specific tolerance, and if there is a match and similarities, the output block will indicate a normal system behaviour. Otherwise, the current values are processed in the second decision step and will be compared with values from weeks or even months ago. In case of similarities, 
the output power will be corrected with a factor that depends by the sun position, according to [7]:

$$
D N I=D N I_{0} * e^{-m * \sum t i}
$$

where

$D N I$ - direct normal irradiance;

$D N I_{0}$ - extra-terrestrial beam irradiance;

$m$ - air mass depending on the sun position in time, means day of the year, hour, etc it can be calculated accurately;

$\sum t i$ is a sum of five coefficients that depend on atmosphere. For simplicity, $\sum t i$ is approximated with a constant coefficient, thus according to [8], the equation (1) becomes:

$$
D N I=D N I_{0} * 0.7^{m^{0.678}}
$$

Considering the output power $(P)$, directly proportional with $D N I$, there is:

- for the historical time, the output power $\left(P_{\text {matched }}\right)$ is:

$$
P_{\text {matched }}=k * D N I_{0} * 0.7^{m \_ \text {matched }} 0.678
$$

- for the present time, the output power $\left(P_{m \_ \text {pres }}\right)$ is:

$$
P_{m \_ \text {pres }}=k * D N I_{0} * 0.7^{m \_p r e s^{0.678}}
$$

where $k$ is a coefficient witch not relevant as we will see below.

Thus, making a ratio between (4) and (3) it results that:

$$
P_{m \_ \text {pres }}=P_{\text {matched }} * \frac{0.7^{m \_ \text {pres }}{ }^{0.678}}{0.7^{m} \text { matched }^{0.678}}
$$

The coefficient for sun position correction is defined as:

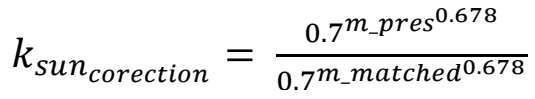

This coefficient is used in the output block where $P_{m \_ \text {pres }}$ will be compared with $P_{\text {present }}$ and, in case of similar values are recorded (including some accepted tolerance) then the system operation is consider as normal.

The other blocks of the Monitoring Engine will compare the current values with historical records from the same season or year (s). In this last case the power will be corrected $k_{\text {sun }_{\text {corection }}}$. In case no similarities are detected between the current values and historical records, then the tolerance value can be decreased, for example if initial match tolerance is 5\%, then it can decrease to $10 \%$, and the entire process is repeated starting with the first decision block. In case no similarities are detected, then alerts are triggered to further investigate the components of the PV system.

The computational resources required by the Monitoring Engine are high in case of the last two decision blocks, thus involving powerful processing technologies. In the following section, an example of the implementation of the proposed method is provided.

\section{Prototyping}

For testing and prototyping the Monitoring Engine, the functional schema presented in Figure 3 can be used.

The other R package from RHadoop suite is rhdfs which provides the connection to HDFS from R. RHIPE package is an R API that can be used with Hadoop, it is similar with RHadoop. An excerpt from the prototype, running MapReduce is provided in Figure 4. Considering Hadoop scalability, the prototyping diagram can be used even in production system for transition from classical monitoring systems to monitoring systems that integrates big data from sensors, as described in Figure 1. 


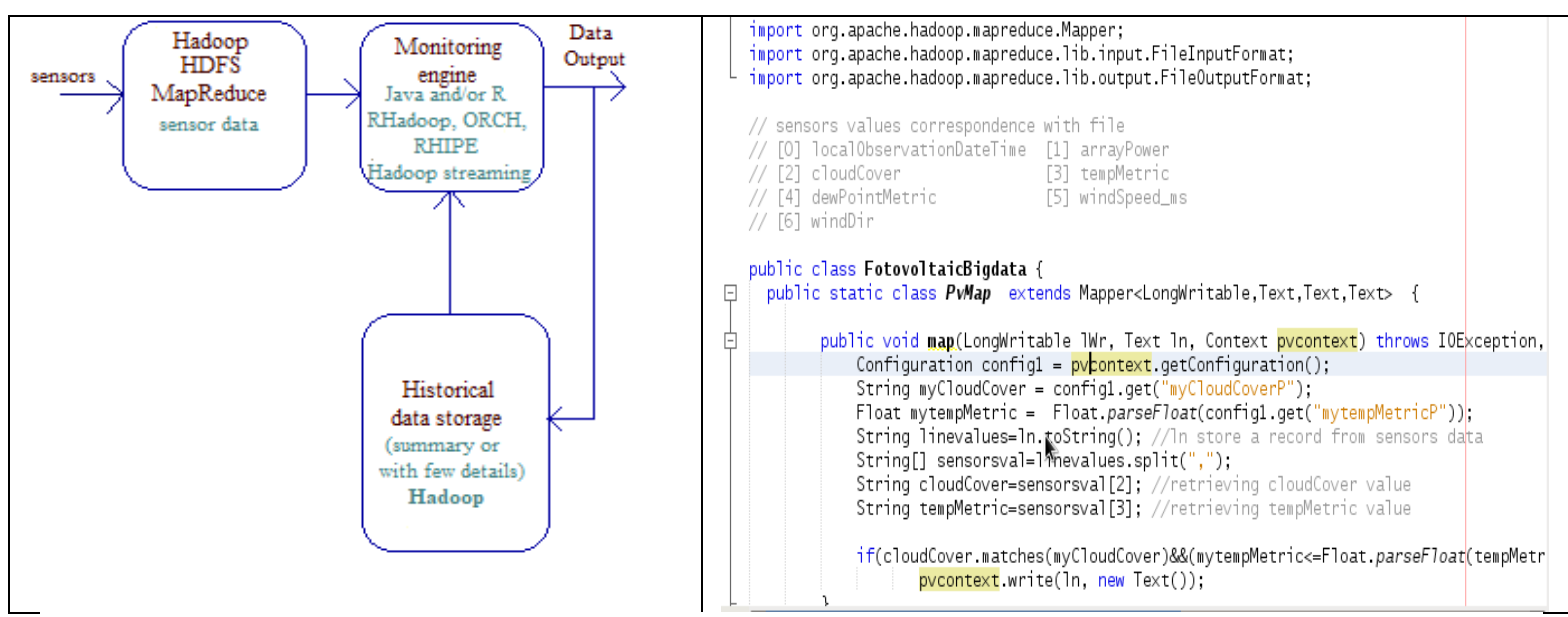

Figure 3. Prototyping the Monitoring Engine and an excerpt from mapreduce java code used for filtering in map method

\begin{tabular}{|c|c|}
\hline 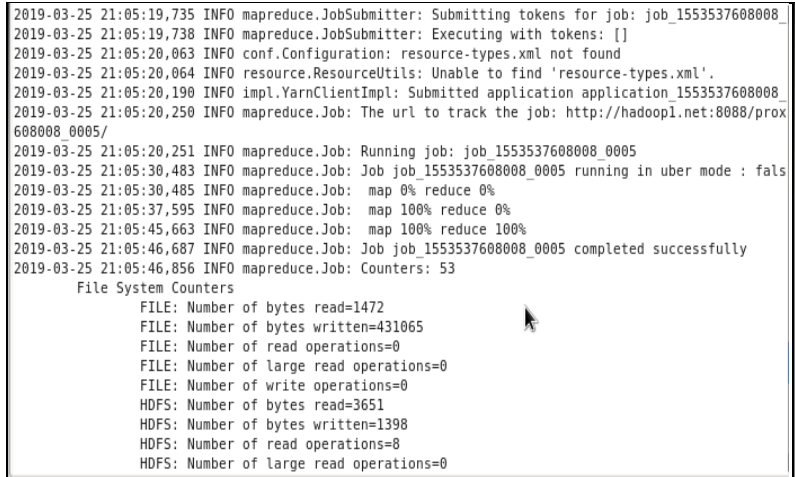 & 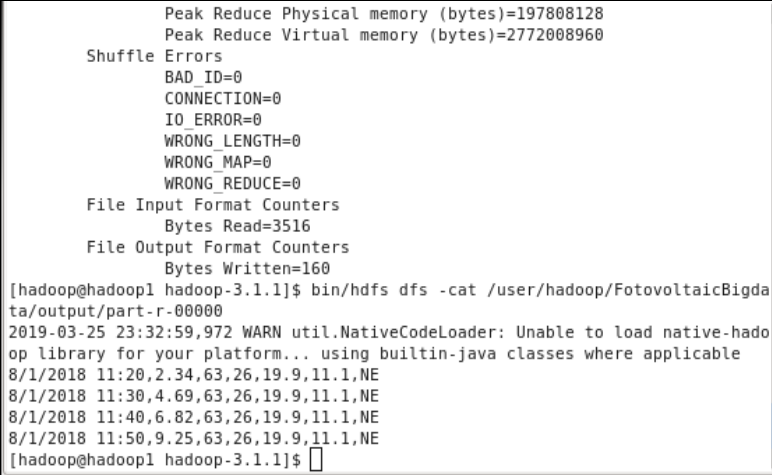 \\
\hline
\end{tabular}

Figure 4. Output excerpt from running MapReduce filtering for cloudCover $=63 \%$ and tempMetric $=26^{\circ} \mathrm{C}$

\section{Conclusions}

Considering different time resolutions for monitoring purposes of the PV systems involves big data technologies for data collection, processing and storage. A big data architecture, including the Monitoring Engine component were proposed considering relevant input parameters for PV generated power such as weather and PV modules /inverters data. The accuracy of the monitoring activity increases in time, by increasing the amount of the historical data stored in the HDFS files. The Monitoring Engine compares the actual measured values with historical records, adjust the generated power in some circumstances and make some estimations regarding the status of the PV system operation. The Monitoring Engine is implemented under the Hadoop ecosystem using Java and R for data processing.

\section{Acknowledgment}

This paper presents the scientific results of the project "Intelligent system for trading on wholesale electricity market" (SMARTRADE), co-financed by the European Regional Development Fund (ERDF), through the Competitiveness Operational Programme (COP) 2014-2020, priority axis 1 - Research, technological development and innovation (RD\&I) to support economic competitiveness and business development, Action 1.1.4 - Attracting highlevel personnel from abroad in order to enhance the RD capacity, contract ID P_37_418, no. 62/05.09.2016, beneficiary: The Bucharest University of Economic Studies. 
www.conferenceie.ase.ro

\section{References}

[1] J. Balfour, M. Shaw, N.B.Nash, Introduction to Photovoltaic System Design, Jones \& Bartlett Learning, November 2011, ISBN: 9781449624682

[2] International Energy Agency, Photovoltaic Power Systems Programme, "Analytical Monitoring of Grid-connected Photovoltaic Systems. Good Practices for Monitoring and Performance Analysis", IEA PVPS Task 13, Subtask 2, Report IEA-PVPS T13-03: 2014, March 2014, ISBN 978-3-906042-18-3

[3] S.Preda, S.-V. Oprea, A.Bâra, A. Belciu (Velicanu), "PV Forecasting Using Support Vector Machine Learning in a Big Data Analytics Context", Symmetry 2018, 10, 748.

[4] T. Rabl, Big Data Stream Processing, Berlin Big Data Center. [Online]. Available:

https://www.scads.de/images/Events/3rdSummerSchool/Talks/TRabl_StreamProcessing.pdf. [Accessed March 2019].

[5] E A. El-Shafeiy, A.I. El-Desouky, “A Big Data Framework for Mining Sensor Data Using Hadoop", Studies in Informatics and Control, vol. 26, no. 3, pp. 365-376, September 2017

[6] J. Kleissl, Solar Energy Forecasting and Resource Assessment, Academic Press, June 2013, ISBN: 9780123977724

[7] T. Stoffel, J. Michalsky, F. Vignola, Solar and Infrared Radiation Measurements, CRC Press, December 2017, ISBN: 9781351833264

[8] Air mass, solar intensity. [Online]. Available: https://en.wikipedia.org/wiki/Air_mass_(solar_energy). [Accessed March 2019].

[9] V. Prajapati, Big Data Analytics with R and Hadoop, Packt Publishing, November 2013, ISBN: 9781782163282

[10] S. Alla, Big Data Analytics with Hadoop 3, Packt Publishing, May 2018, ISBN: 9781788628846

[11] T. Plunkett, M. Hornick, Using R to Unlock the Value of Big Data: Big Data Analytics with Oracle R Enterprise and Oracle R Connector for Hadoop, McGraw-Hill, Oracle Press, June 2013, ISBN: 9780071824385 